\title{
A geometrical interpretation of the inverse matrix
}

\section{Yanping Zhou* and Binwu He}

${ }^{*}$ Correspondence:

zhouyp@i.shu.edu.cn

Department of Mathematics,

Shanghai University, Shanghai,

200444, China

\section{Springer}

\begin{abstract}
Utilizing a new method to structure parallellotopes, a geometrical interpretation of the inverse matrix is given, which includes the generalized inverse of full column rank or a full row rank matrices. Further, some relational volume formulas of parallellotopes are established.
\end{abstract}

MSC: $15 \mathrm{~A} 15 ; 52 \mathrm{~A} 20$

Keywords: parallellotope; inverse matrix; generalized inverse

\section{Introduction and notations}

Let $\mathbb{R}^{n}$ denote an $n$-dimensional real Euclidean vector space, for a nonzero $n \times 1$ vector $x \in \mathbb{R}^{n}$, the generalized inverse of $x$, denoted by $x^{+}$, has the geometrical interpretation that $x^{T}$ is divided by $\|x\|^{2}$, that is, $x^{+}=x^{T} /\|x\|^{2}$, where $x^{T}$ is the transpose of $x$ (see [1]). A natural question is whether a similar geometrical interpretation holds for the inverse of a matrix.

In this paper, using a new method to structure a $m$-dimensional parallellotope, the geometrical interpretation of the inverse matrix and the generalized inverse of a matrix with full column rank or full row rank are given.

Let $\left[z_{1}, z_{2}, \ldots, z_{m}\right]$ be the $m$-dimensional parallellotope with $m$ linearly independent vectors $z_{1}, z_{2}, \ldots, z_{m}$ as its edge vectors, i.e.,

$$
\left[z_{1}, z_{2}, \ldots, z_{m}\right]=\left\{z \in \mathbb{R}^{n} \mid t_{1} z_{1}+\cdots+t_{m} z_{m}, t_{i} \in[0,1], i=1,2, \ldots, m\right\}
$$

$\left[z_{1}, \ldots, z_{i-1}, z_{i+1}, \ldots, z_{m}\right]$ denotes the facets of the $m$-parallellotope $\left[z_{1}, z_{2}, \ldots, z_{m}\right]$ for an (m-1)-hyperplane,

$$
\mathcal{H}_{i}=\operatorname{span}\left\{z_{1}, \ldots, z_{i-1}, z_{i+1}, \ldots, z_{m}\right\}
$$

$z_{i}$ is the altitude vector on facet $\left[z_{1}, \ldots, z_{i-1}, z_{i+1}, \ldots, z_{m}\right]$ (see $\left.[2,3]\right)$ with the orthogonal component of $z_{i}$ with respect to $\mathcal{H}_{i}$. If $\left[z_{1}, z_{2}, \ldots, z_{m}\right]^{*}$ denotes the $m$-parallellotope constructed by $m$ linearly independent vectors $z_{1}, z_{2}, \ldots, z_{m}$ as its altitude vectors, then we will show that there exist $z_{1}^{*}, z_{2}^{*}, \ldots, z_{m}^{*}$, exclusive such that

$$
\left[z_{1}, z_{2}, \ldots, z_{m}\right]^{*}=\left[z_{1}^{*}, z_{2}^{*}, \ldots, z_{m}^{*}\right]
$$

(c) 2016 Zhou and He. This article is distributed under the terms of the Creative Commons Attribution 4.0 International License (http://creativecommons.org/licenses/by/4.0/), which permits unrestricted use, distribution, and reproduction in any medium, provided you give appropriate credit to the original author(s) and the source, provide a link to the Creative Commons license, and indicate if changes were made. 


\section{Main results}

Our main results are the following theorems.

Theorem 2.1 If $M$ is a matrix with full row (column) rank and $z_{1}, z_{2}, \ldots, z_{m}$ is its row (column) vectors, then the right (left) inverse of the matrix $M$ is the matrix whose column (row) vectors are

$$
\frac{z_{1}^{*}}{\left\|z_{1}\right\|^{2}}, \frac{z_{2}^{*}}{\left\|z_{2}\right\|^{2}}, \ldots, \frac{z_{m}^{*}}{\left\|z_{m}\right\|^{2}},
$$

where $z_{1}^{*}, z_{2}^{*}, \ldots, z_{m}^{*}$ are $m$ edge vectors of the $m$-parallellotope $\left[z_{1}, z_{2}, \ldots, z_{m}\right]^{*}$.

Corollary 2.2 If $M$ is nonsingular $n \times n$ matrix and $z_{1}, z_{2}, \ldots, z_{n}$ is its row (column) vectors, then the inverse of the matrix $M$ is the matrix whose column (row) vectors are

$$
\frac{z_{1}^{*}}{\left\|z_{1}\right\|^{2}}, \frac{z_{1}^{*}}{\left\|z_{1}\right\|^{2}}, \ldots, \frac{z_{n}^{*}}{\left\|z_{n}\right\|^{2}},
$$

where $z_{1}^{*}, z_{2}^{*}, \ldots, z_{n}^{*}$ are $n$ edge vectors of the $n$-parallellotope $\left[z_{1}, z_{2}, \ldots, z_{n}\right]^{*}$.

We may say roughly if the $\left[z_{1}, z_{2}, \ldots, z_{m}\right]\left(z_{1}, z_{2}, \ldots, z_{m}\right.$ as edge vectors) is the geometrical interpretation of the matrix $M$, then $\left[z_{1}, z_{2}, \ldots, z_{m}\right]^{*}\left(z_{1}, z_{2}, \ldots, z_{m}\right.$ as altitude vectors $)$ is one of the $M^{-1}$.

We list some basic facts to state the following theorems.

We write $L(i)$, for the linear subspace spanned by $z_{1}, z_{2}, \ldots, z_{i}, z_{i} \in \mathbb{R}^{n}(1 \leq i \leq n)$. Let $\langle z, L\rangle$ be the angle between vector $z$ and linear subspace $L$, where if $z \notin L$, then $\langle z, L\rangle$ is the angle between $z$ and the orthogonal projection of $z$ on $L$, denoted by $\left.z\right|_{L}$, i.e., $\left.z\right|_{L}=$ $\left(\left(L^{\perp}+x\right) \cap L\right)$. If $z \in L$, then $\langle z, L\rangle=0$.

Theorem 2.3 Suppose $y_{1}, y_{2}, \ldots, y_{n}$ are $n$ row vectors of the matrix $M$, and $z_{1}, z_{2}, \ldots, z_{n}$ are column vectors of the matrix $M^{-1}$,

(1) if $\left\|y_{i}\right\| \rightarrow 0$, then $\left\|z_{i}\right\| \rightarrow+\infty$;

(2) if $\left\langle\hat{y_{i}, L}(i-1)\right\rangle \rightarrow 0$, then there is $k(1 \leq k \leq n)$ such that $\left\|z_{k}\right\| \rightarrow+\infty$.

Theorem 2.3 will be required in the study of matrix disturbances (see [4-6]).

Utilizing the geometrical interpretation of the inverse matrix, we have the following relational volume formulas of parallellotopes for the $n \times n$ real matrices $M, N$.

Theorem 2.4 Let $\left[z_{1}, z_{2}, \ldots, z_{n}\right]^{* *}$ be the parallellotope structured by the edge vectors of $\left[z_{1}, z_{2}, \ldots, z_{n}\right]^{*}$ as altitude vectors. Then

$$
\begin{aligned}
& \operatorname{vol}\left(\left[z_{1}, z_{2}, \ldots, z_{n}\right]^{*}\right) \cdot \operatorname{vol}\left(\left[z_{1}, z_{2}, \ldots, z_{n}\right]\right)=\left(\prod_{i=1}^{n}\left\|z_{i}\right\|\right)^{2}, \\
& \operatorname{vol}\left(\left[z_{1}, z_{2}, \ldots, z_{n}\right]^{* *}\right) / \operatorname{vol}\left(\left[z_{1}, x_{2}, \ldots, z_{n}\right]\right)=\left(\prod_{i=1}^{n}\left\|z_{i}^{*}\right\| /\left\|z_{i}\right\|\right)^{2},
\end{aligned}
$$

where $\operatorname{vol}\left(\left[z_{1}, \ldots, z_{n}\right]\right)$ denotes the volume of the parallellotope $\left[z_{1}, \ldots, z_{n}\right]$.

The proofs of the theorems will be given in Section 3. 


\section{Proofs of the theorems}

Given $m$ linearly independent vectors $z_{1}, z_{2}, \ldots, z_{m}$ in $\mathbb{R}^{n}$, if we structure an $m$-parallellotope $\left[z_{1}, z_{2}, \ldots, z_{m}\right]$ by them as edge vectors, then $\left[z_{1}, z_{2}, \ldots, z_{m}\right]$ has $m$ linearly independent altitude vectors. Conversely, for any given $m$ linearly independent vectors $z_{1}, z_{2}, \ldots, z_{m}$, can we structure an $m$-parallellotope by them as $m$ altitude vectors? The following lemma gives an affirmative answer.

Lemma 3.1 If $\left\{z_{1}, z_{2}, \ldots, z_{m}\right\}(m \geq 2)$ is a given set of linearly independent vectors in $\mathbb{R}^{n}$, then there is an m-parallellotope $\left[z_{1}, z_{2}, \ldots, z_{m}\right]^{*}$ whose $m$ altitude vectors are $z_{1}, z_{2}, \ldots, z_{m}$.

Proof If $z_{1}, z_{2}, \ldots, z_{m}$ are linearly independent, then we have $m$ linear functionals $g_{1}, g_{2}, \ldots$, $g_{m}$ such that

$$
g_{j}\left(z_{i}\right)=\delta_{i j}\left\|z_{i}\right\|^{2}, \quad i, j=1,2, \ldots, m,
$$

where $\delta_{i j}$ is the Kronecker delta symbol.

From Riesz's representation theorem for the linear functional, we get $z_{1}^{*}, z_{2}^{*}, \ldots, z_{m}^{*}$ such that

$$
\left\langle z_{i}, z_{j}^{*}\right\rangle=\delta_{i j}\left\|z_{i}\right\|^{2}, \quad i, j=1,2, \ldots, m,
$$

where $\langle$,$\rangle is the ordinary inner product in \mathbb{R}^{n}$.

Further, let

$$
\sum_{j=1}^{m} \alpha_{j} z_{j}^{*}=0, \quad \alpha_{j} \in \mathbb{R},
$$

by

$$
0=\left\langle z_{i}, \sum_{j=1}^{m} \alpha_{j} z_{j}^{*}\right\rangle=\alpha_{i}\left\|z_{i}\right\|^{2},
$$

we have $\alpha_{i}=0, i=1,2, \ldots, m$. This shows that $z_{1}^{*}, z_{2}^{*}, \ldots, z_{m}^{*}$ are linearly independent.

Now, we prove that $z_{1}, z_{2}, \ldots, z_{m}$ are altitude vectors of the $m$-parallellotope $\left[z_{1}^{*}, z_{2}^{*}, \ldots\right.$, $\left.z_{m}^{*}\right]$ (the edge vectors of $\left[z_{1}^{*}, z_{2}^{*}, \ldots, z_{m}^{*}\right]$ are $z_{1}^{*}, z_{2}^{*}, \ldots, z_{m}^{*}$ ).

Suppose that $\left[z_{1}^{*}, z_{2}^{*}, \ldots, z_{i-1}^{*}, z_{i+1}^{*}, \ldots, z_{m}^{*}\right]$ are the facets of $\left[z_{1}^{*}, z_{2}^{*}, \ldots, z_{m}^{*}\right]$. From $z_{i} \perp z_{j}^{*}$ $(j \neq i)$, we have

$$
z_{i} \perp\left[z_{1}^{*}, z_{2}^{*}, \ldots, z_{i-1}^{*}, z_{i+1}^{*}, \ldots, z_{m}^{*}\right]
$$

Thus, $z_{1}, z_{2}, \ldots, z_{m}$ are altitude vectors of $\left[z_{1}^{*}, z_{2}^{*}, \ldots, z_{m}^{*}\right]$, i.e.,

$$
\left[z_{1}, z_{2}, \ldots, z_{m}\right]^{*}=\left[z_{1}^{*}, z_{2}^{*}, \ldots, z_{m}^{*}\right]
$$

This yields the desired $m$-parallellotope $\left[z_{1}, z_{2}, \ldots, z_{m}\right]^{*}$. 
Proof of Theorem 2.1 For a given $m \times n$ matrix full row $\operatorname{rank} M=\left(c_{i j}\right)_{m \times n}$, let

$$
z_{i}=\left(c_{i 1}, c_{i 2}, \ldots, c_{i n}\right), \quad i=1,2, \ldots, m
$$

By Lemma 3.1, we have an unique vector set $\left\{z_{1}^{*}, z_{2}^{*}, \ldots, z_{m}^{*}\right\}$ such that

$$
\left\langle z_{i}, z_{j}^{*}\right\rangle=\delta_{i j}\left\|z_{i}\right\|^{2}, \quad i=1,2, \ldots, m ; j=1,2, \ldots, n,
$$

i.e.,

$$
\left\langle z_{i}, \frac{z_{j}^{*}}{\left\|z_{i}\right\|^{2}}\right\rangle=\delta_{i j}, \quad i=1,2, \ldots, m ; j=1,2, \ldots, n,
$$

and $z_{1}^{*}, z_{2}^{*}, \ldots, z_{m}^{*}$ are $m$ edge vectors of the parallellotope $\left[z_{1}, z_{2}, \ldots, z_{m}\right]^{*}$.

Suppose

$$
d_{i}=\frac{z_{i}^{*}}{\left\|z_{i}\right\|^{2}}, \quad i=1,2, \ldots, m
$$

and

$$
N=\left(d_{1}, d_{2}, \ldots, d_{m}\right)
$$

It follows from (3.3) that

$$
M N=\left(\begin{array}{c}
z_{1} \\
z_{2} \\
\vdots \\
z_{m}
\end{array}\right)\left(d_{1}, d_{2}, \ldots, d_{m}\right)=\left(\begin{array}{ccc}
1 & & 0 \\
& \ddots & \\
0 & & 1
\end{array}\right) .
$$

Thus, the matrix $N$ is the inverse of the matrix $M$, and the column vectors $d_{1}, d_{2}, \ldots, d_{m}$ of the matrix $N$ are the edge vectors of $\left[z_{1}, z_{2}, \ldots, z_{m}\right]^{*}$ divided by $\left\|z_{1}\right\|^{2},\left\|z_{2}\right\|^{2}, \ldots,\left\|z_{m}\right\|^{2}$, respectively.

Together with Theorem 2.1 and taking $M$ for an $n \times n$ matrix with full rank, we have Corollary 2.2.

Here, we will complete the proof of Theorem 2.3. The following lemma will be required.

Lemma 3.2 For $L(i)$ the linear subspace spanned by $z_{1}, z_{2}, \ldots, z_{i}, i=1,2, \ldots, m(\leq n)$, if $\operatorname{vol}\left(\left[z_{1}, z_{2}, \ldots, z_{m}\right]\right)$ is the volume of the parallellotope $\left[z_{1}, z_{2}, \ldots, z_{m}\right]$ (see [7]), we have

$$
\operatorname{vol}\left(\left[z_{1}, z_{2}, \ldots, z_{m}\right]\right)=\prod_{i=1}^{m}\left\|z_{i}\right\| \cdot \prod_{i=2}^{m} \sin \left\langle\hat{z_{i}, L(i-1)}\right\rangle .
$$

Proof Assume that $h_{i}, p_{i}$ are the orthogonal component and orthogonal projection of $z_{i}$ with respect to $L(i-1)$, respectively $\left(i=2, \ldots, m, h_{1}=z_{1}, p_{1}=0\right)$. Since $\left\|z_{i}\right\| \cos \left\langle z_{i}, p_{i}\right\rangle=$ $\left\|p_{i}\right\|$, we have

$$
\cos \left\langle z_{i}, L(i-1)\right\rangle=\frac{\left\langle z_{i}, p_{i}\right\rangle}{\left\|z_{i}\right\|\left\|p_{i}\right\|}=\frac{\left\langle p_{i}, p_{i}\right\rangle}{\left\|z_{i}\right\|\left\|p_{i}\right\|}=\frac{p_{i}}{\left\|z_{i}\right\|} .
$$


By $\left\|z_{i}\right\|^{2}=\left\|p_{i}\right\|^{2}+\left\|h_{i}\right\|^{2}$, it follows that

$$
\left\|h_{i}\right\|=\sqrt{\left\|z_{i}\right\|^{2}-\left\|p_{i}\right\|^{2}}=\left\|z_{i}\right\| \sin \left\langle z_{i}, L(i-1)\right\rangle .
$$

From the definition of the volume of the parallellotope, we get (see [7-9])

$$
\operatorname{vol}\left(\left[z_{1}, z_{2}, \ldots, z_{m}\right]\right)=\prod_{i=1}^{m}\left\|h_{i}\right\|=\prod_{i=1}^{m}\left\|z_{i}\right\| \cdot \prod_{i=2}^{m} \sin \left\langle z_{i}, L(i-1)\right\rangle
$$

The proof of Lemma 3.2 is completed.

Proof of Theorem 2.3 From Theorem 2.1, it follows that

$$
\left(\begin{array}{c}
y_{1} \\
y_{2} \\
\vdots \\
y_{n}
\end{array}\right)\left(z_{1}, z_{2}, \ldots, z_{n}\right)=\left(\begin{array}{ccc}
\left\langle y_{1}, z_{1}\right\rangle & & 0 \\
& \ddots & \\
0 & & \left\langle y_{1}, z_{1}\right\rangle
\end{array}\right)=\left(\begin{array}{ccc}
1 & & 0 \\
& \ddots & \\
0 & & 1
\end{array}\right)
$$

i.e.,

$$
\left\langle y_{i}, z_{i}\right\rangle=1, \quad i=1,2, \ldots, n
$$

It follows from the Cauchy inequality that

$$
1=\left|\left\langle y_{i}, z_{i}\right\rangle\right| \leq\left\|y_{i}\right\|\left\|z_{i}\right\|
$$

Thus the assertion (1) holds.

Let $\left\{y_{1}, y_{2}, \ldots, y_{n}\right\}$ and $\left\{z_{1}, z_{2}, \ldots, z_{n}\right\}$ in Lemma 3.2. From (3.7), we get

$$
\left(\prod_{i=1}^{n}\left\|y_{i}\right\| \cdot \prod_{i=1}^{n} \sin \left\langle\hat{y_{i}, L(i-1)}\right\rangle\right) \cdot\left(\prod_{j=1}^{n}\left\|z_{j}\right\| \cdot \prod_{j=1}^{n} \sin \left\langle\hat{z_{j}, L(j-1)}\right\rangle\right)=1 .
$$

From

$$
0 \leq \mid \prod_{j=1}^{n} \sin \left\langle\hat{\left.y_{j}, L(j-1)\right\rangle}\right| \leq 1
$$

and

$$
\prod_{i=1}^{n}\left\|y_{i}\right\| \leq G
$$

the assertion (2) is given. 
Proof of Theorem 2.4 Together with Theorem 2.1, we get

$$
\left(\begin{array}{c}
\frac{z_{1}^{*}}{\left\|z_{1}\right\|^{2}} \\
\frac{z_{2}^{*}}{\left\|z_{2}\right\|^{2}} \\
\vdots \\
\frac{z_{n}^{*}}{\left\|z_{n}\right\|^{2}}
\end{array}\right)\left(z_{1}, z_{2}, \ldots, z_{n}\right)=\left(\begin{array}{ccc}
1 & & 0 \\
& \ddots & \\
0 & & 1
\end{array}\right)
$$

Thus

$$
\begin{aligned}
& \operatorname{det}\left(\left(\begin{array}{c}
\frac{z_{1}^{*}}{\left\|z_{1}\right\|^{2}} \\
\frac{z_{2}^{*}}{\left\|z_{2}\right\|^{2}} \\
\vdots \\
\frac{z_{n}^{*}}{\left\|z_{n}\right\|^{2}}
\end{array}\right)\left(z_{1}, z_{2}, \ldots, z_{n}\right)\right)=1 \\
& \operatorname{det}\left(\begin{array}{c}
z_{1}^{*} \\
z_{2}^{*} \\
\vdots \\
z_{n}^{*}
\end{array}\right) \cdot \operatorname{det}\left(z_{1}, z_{2}, \ldots, z_{n}\right)=\left(\prod_{i=1}^{n}\left\|z_{i}\right\|\right)^{2} \text {. }
\end{aligned}
$$

From

$$
\left[x_{1}, x_{2}, \ldots, x_{n}\right]^{*}=\left[z_{1}^{*}, z_{2}^{*}, \ldots, z_{n}^{*}\right]
$$

and the definition of the volume of parallellotopes, the equality (2.1) holds.

Assume that $\left\{z_{1}^{* *}, z_{2}^{* *}, \ldots, z_{n}^{* *}\right\}$ is a set of the edge vectors of $\left[z_{1}, z_{2}, \ldots, z_{n}\right]^{* *}$. Together with Theorem 2.1, we get

$$
\left(\begin{array}{c}
z_{1}^{*} \\
z_{2}^{*} \\
\vdots \\
z_{n}^{*}
\end{array}\right)\left(\frac{z_{1}^{* *}}{\left\|z_{1}^{*}\right\|^{2}}, \frac{z_{2}^{* *}}{\left\|z_{2}^{*}\right\|^{2}}, \vdots, \frac{z_{n}^{* *}}{\left\|z_{n}^{*}\right\|^{2}}\right)=\left(\begin{array}{ccc}
1 & & 0 \\
& \ddots & \\
0 & & 1
\end{array}\right)
$$

If follows from (3.10) that

$$
\operatorname{det}\left(\begin{array}{c}
z_{1}^{*} \\
z_{2}^{*} \\
\vdots \\
z_{n}^{*}
\end{array}\right) \cdot \operatorname{det}\left(z_{1}^{* *}, z_{2}^{* *}, \ldots, z_{n}^{* *}\right)=\left(\prod_{i=1}^{n}\left\|z_{i}\right\|\right)^{2}
$$

Thus

$$
\operatorname{vol}\left(\left[z_{1}, z_{2}, \ldots, z_{n}\right]^{*}\right) \cdot \operatorname{vol}\left(\left[z_{1}, z_{2}, \ldots, z_{n}\right]^{* *}\right)=\left(\prod_{i=1}^{n}\left\|z_{i}^{*}\right\|\right)^{2}
$$

Taking together (2.1) and (3.11), the equality (2.2) holds. 
For $\left\{z_{1}, z_{2}, \ldots, z_{n}\right\}$, from Lemma 3.1, $\left[z_{1}, z_{2}, \ldots, z_{n}\right]^{*}$ is structured by them as altitude vectors. Denote $\left[z_{1}, z_{2}, \ldots, z_{n}\right]^{*}$ by $z_{1}^{*}, z_{2}^{*}, \ldots, z_{n}^{*}$.

Let

$$
\left[z_{1}, z_{2}, \ldots, z_{n}\right]^{* *}=\left[z_{1}^{*}, z_{2}^{*}, \ldots, z_{n}^{*}\right]^{*}
$$

Thus Theorem 2.4 denotes the relationship of volumes about $\left[z_{1}, z_{2}, \ldots, z_{n}\right],\left[z_{1}, z_{2}, \ldots, z_{n}\right]^{*}$, and $\left[z_{1}, z_{2}, \ldots, z_{n}\right]^{* *}$.

Remark 1 By (3.10), we get

$$
\left(\begin{array}{c}
\frac{z_{1}^{*}}{\left\|z_{1}\right\|^{2}} \\
\frac{z_{2}^{*}}{\left\|z_{2}\right\|^{2}} \\
\vdots \\
\frac{z_{n}^{*}}{\left\|z_{n}\right\|^{2}}
\end{array}\right)\left(\frac{\left\|z_{1}\right\|^{2}}{\left\|z_{1}^{*}\right\|^{2}} z_{1}^{* *}, \frac{\left\|z_{2}\right\|^{2}}{\left\|z_{2}^{*}\right\|^{2}} z_{2}^{* *}, \vdots, \frac{\left\|z_{n}\right\|^{2}}{\left\|z_{n}^{*}\right\|^{2}} z_{n}^{* *}\right)=\left(\begin{array}{ccc}
1 & & 0 \\
& \ddots & \\
0 & & 1
\end{array}\right)
$$

From (3.9) and (3.12), we see that

$$
z_{i}^{* *}=\frac{\left\|z_{i}^{*}\right\|^{2}}{\left\|z_{i}\right\|^{2}} z_{i}, \quad i=1,2, \ldots, n .
$$

By (3.13), we can see that $\left[z_{1}, z_{2}, \ldots, z_{n}\right]^{* *}$ and $\left[z_{1}, z_{2}, \ldots, z_{n}\right]$ are two parallellotopes and their edge vectors are of the same direction.

Competing interests

The authors declare that they have no competing interests.

\section{Authors' contributions}

All authors contributed equally to the writing of this paper. All authors read and approved the final manuscript.

\section{Acknowledgements}

The authors would like to acknowledge the support from the National Natural Science Foundation of China (11371239).

Received: 26 April 2016 Accepted: 5 October 2016 Published online: 19 October 2016

\section{References}

1. Perose, A: A generalized inverse for matrices. Proc. Camb. Philos. Soc. 51, 406-413 (1955)

2. Berger, M: Geometry I. Springer, New York (1987)

3. Veljan, D: The sine theorem and inequalities for volume of simplices and determinants. Linear Algebra Appl. 219, 79-91 (1995)

4. Horn, RA, Johnson, CR: Matrix Analysis. Cambridge University Press, Cambridge (1988)

5. Golub, GH, Van Loan, CF: Matrix Computations, 2nd edn. Johns Hopkings University Press, Baltimore (1989)

6. Golub, GH, Van Loan, CF: Matrix Computations, 4th edn. Johns Hopkings University Press, Baltimore (2013)

7. Ben-Israel, A: A volume associated with $m \times n$ matrices. Linear Algebra Appl. 167, 87-111 (1992)

8. Ben-Israel, A: An application of the matrix volume in probability. Linear Algebra Appl. 321, 9-25 (2000)

9. Ben-Israel, A: The change of variables formula using matrix volume. SIAM J. Matrix Anal. Appl. 21, 300-312 (1999) 